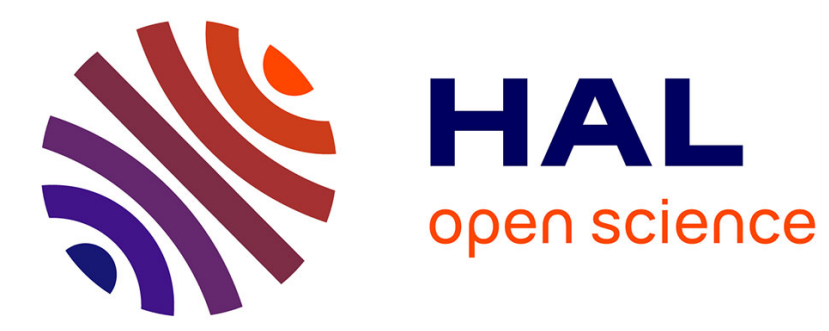

\title{
Prolégomènes à la constitution de Dieu
}

Emmanuel Housset

\section{To cite this version:}

Emmanuel Housset. Prolégomènes à la constitution de Dieu. Transversalités, 2014, Identités, communautés, institutions, 2 (130), pp.151-175. 10.3917/trans.130.0151 . hal-02145544

HAL Id: hal-02145544

https://hal-normandie-univ.archives-ouvertes.fr/hal-02145544

Submitted on 3 Jun 2019

HAL is a multi-disciplinary open access archive for the deposit and dissemination of scientific research documents, whether they are published or not. The documents may come from teaching and research institutions in France or abroad, or from public or private research centers
L'archive ouverte pluridisciplinaire HAL, est destinée au dépôt et à la diffusion de documents scientifiques de niveau recherche, publiés ou non, émanant des établissements d'enseignement et de recherche français ou étrangers, des laboratoires publics ou privés.

\section{(ㄷ)(1)}

Distributed under a Creative Commons Attribution| 4.0 International License 
Emmanuel Housset

Identité et Subjectivité

Unicaen

\section{Prolégomènes à la constitution de Dieu ${ }^{1}$}

Il serait légitime de se demander pourquoi écrire un livre sur Husserl et l'idée de Dieu ${ }^{2}$, même dans la certitude que Husserl est un philosophe majeur du vingtième siècle dont la connaissance est indispensable pour toute pensée qui veut trouver son avenir dans la fidélité au phénomène. Force est de constater que Husserl parle relativement peu de Dieu dans ses grandes œuvres et que la question n'est que rarement abordée directement dans les manuscrits de recherche, même si, et c'est sans doute là déjà un signe, le problème de Dieu n'est jamais totalement absent de sa pensée et resurgit de façon régulière et souvent à des moments importants. Certes, la fréquence et la longueur des analyses ne sont pas en elles-mêmes des critères suffisants, et l'histoire de la métaphysique peut montrer que certaines thèses qui n'apparaissent qu'une seule fois dans un corpus ont pu faire époque et demeurent ce qui anime le présent de la pensée tant il y a en elles de nouveauté irréductible. Cela dit les analyses husserliennes sur Dieu comme sujet idéal de la connaissance n'ont pas ce caractère d'événement originaire de la pensée et ne viennent que confirmer ce qui a déjà été mis en

\footnotetext{
${ }^{1}$ Il me faut remercier chaleureusement le père Philippe Capelle pour m'avoir demandé ce livre pour sa collection Philosophie et théologie aux Editions du Cerf, Emmanuel Falque doyen de la faculté de philosophie pour avoir voulu cette séance autour de mon livre à l'Institut catholique de Paris, Jérôme de Gramont pour avoir accepté de répondre à ce livre et Dominique Pradelle pour être venu participer à cette rencontre autour de Husserl. Il aurait peut-être semblé étrange à Husserl de voir ses analyses discutées dans un institut catholique, lui qui revendiquait un athéisme méthodologique, mais le siècle dans lequel nous parlons n'est plus le même et du fait d'un antichristianisme prenant des formes nouvelles le seul fait de parler en philosophe de Dieu est devenu suspect. A cet égard la faculté de philosophie de l'Institut Catholique de Paris, où j'ai eu l'honneur de donner un cours de phénoménologie pendant quatre années, est aujourd'hui, non pas une citadelle assiégée, mais véritablement un espace de liberté dans lequel l'analyse phénoménologique peut se déployer dans toute sa fécondité. Je tenais à la remercier de porter, sans restriction, le sens de l'universel.

2 Afin de ne pas redoubler les analyses réalisées dans Husserl et l'idée de Dieu, il sera fait peu de citations de Husserl dans cet exposé. Pour la bibliographie, déjà abondante sur cette question, je renvoie également à mon livre.
} 
évidence de la phénoménalité de l'objet. En outre, bien d'autres philosophes, à commencer par Kant lui-même, ont pu montrer, et d'une manière bien plus précise et développée, en quoi le Dieu des philosophes libère des dieux du paganisme et peut ainsi reconduire philosophie et religion à leur objet respectif dans la conscience de leur limitation réciproque et de leur confrontation nécessaire. Comment un idéalisme transcendantal pourrait-il aller au-delà de Kant ? Une question analogue se pose avec Descartes : dans bien des passages Husserl est très brutal sur la troisième des Méditations métaphysiques, mais il aurait pu remarquer ${ }^{3}$ qu'en refusant par avance toute idée d'un sujet constituant et en comprenant l'entendement comme passif, Descartes peut, lui, montrer que Dieu n'est pas une idée en avant du sujet, mais la source de la lumière naturelle, ce qui est bien sûr impossible pour une égologie transcendantale. De plus, toute l'analyse intentionnelle de Husserl est méthodologiquement athée et semble dès lors fermer la porte à toute étude des rapports entre philosophie et religion. La thèse cartésienne selon laquelle l'athéisme est incompatible avec la vraie science est inaudible pour Husserl. La question se pose donc bien : pourquoi cet homme des Lumières au milieu des violences du XXème siècle qui non seulement parle peu de Dieu, mais qui en outre semble ne pas pouvoir en parler, peut-il de façon programmatique esquisser une théologie phénoménologique ? Enfin, quel est le sens d'une telle théologie phénoménologique qui laisse totalement de côté toute la théologie révélée, mais également tous les textes proprement théologiques, pour substituer à la théologie existante avec son histoire et ses méthodes, une sorte de «théologie pure »? Autrement dit quel est le sens d'une telle purification philosophique de Dieu qui prétend élucider l'idée de Dieu sans s'installer dans les énoncés proprement théologiques ? N'est-ce pas parler de la théologie de l'extérieur, c'est-à-dire sans la pratiquer, sans être à l'écoute de la Parole?

Les données biographiques, aussi intéressantes soient-elles, ne permettent pas d'élaborer cette question, et doivent être laissées de côté, car elles n'éclairent pas l'objet de l'étude. Il est donc nécessaire de se demander, dans une lecture purement immanente des textes, pourquoi Husserl parle de Dieu en dehors de tout projet d'une philosophie chrétienne et sans vouloir, au moins de façon directe, s'expliquer avec la Bible. Paradoxalement cette distance assumée est peut-être la marque d'une confrontation réelle, et sans doute assez douloureuse, avec le christianisme. Selon Husserl, le philosophe est un universitaire animé par l'exigence

\footnotetext{
${ }^{3}$ Je remercie madame Boudot d'avoir attiré mon attention sur ce point et d'avoir ainsi formulé une objection cartésienne aux analyses de Husserl. Effectivement Husserl est beaucoup moins apte à s'approcher du Dieu de la religion que Descartes puisque sa conception du sujet autonome le contraint à ne voir en Dieu qu'une idée. Néanmoins l'eidos de la subjectivité transcendantale est sans doute plus complexe qu'on ne le pense et surtout la question est ailleurs pour Husserl qui se contente d'élucider le sens de Dieu pour une conscience.
} 
absolue de science rigoureuse et qui ne négocie pas l'autonomie de la pensée, puisqu'il est idéalement, de ce point de vue, l'archonte de l'humanité, mais depuis cette hauteur la voix de celui qui crie dans le désert n'est pas nécessairement devenue inaudible. D'une manière très étonnante, et d'autant plus forte qu'elle est discrète, l'auteur des Recherches logiques peut donner au lecteur que nous sommes d'assister à ce combat de géants entre parole philosophique et parole biblique, entre les amis des formes et les enfants de Dieu.

Il y a alors plusieurs « raisons » de se laisser enseigner parce que l'œuvre de Husserl montre de la phénoménalité de Dieu.

\section{Une interrogation historique.}

Une raison historique en premier lieu, puisqu'il est indispensable du point de vue de la genèse des concepts de comprendre comment Husserl reprend et modifie l'analyse kantienne de Dieu comme Idée de la raison, sans pour autant écrire une «religion dans les limites de la simples raison ». La méthode adoptée consiste de façon délibérée à lire Husserl par la fin, c’està-dire en partant de La Crises des sciences européennes et la phénoménologie transcendantale pour remonter jusqu'aux Recherches logiques, et donc sans présupposer de rupture dans l'œuvre de Husserl, puisque l'idéalisation est un thème absolument continu. Ce chemin volontairement plus téléologique que génétique cherche à mettre en lumière le concept de Dieu tel qu'il finit par se dégager à la fin de la vie intellectuelle de Husserl afin de mieux saisir les étapes de son évolution, c'est-à-dire aussi bien les continuités que les modifications. Cela n'enlève rien aux mérites d'une étude pas à pas de l'histoire d'un concept chez un auteur, mais sans l'analyse du phénomène de Dieu selon Husserl, il est impossible d'écrire une histoire du concept de Dieu dans la pensée de Husserl. En philosophie il n'y a d'histoire que philosophique et toute simple histoire des faits n'est pas une histoire. Pour décrire la naissance de la question de Dieu dans la philosophie de Husserl, il n'est pas possible de se passer de la lumière de ce à quoi cette naissance conduit, ce qui ne signifie pas affirmer que tout était déjà là au commencement.

Du point de vue de l'historicité de la philosophie, il est également important de comprendre pourquoi Husserl est, sans le vouloir, l'initiateur de toute une phénoménologie de la religion et de toute une étude de la phénoménalité de Dieu, qui vont bien au-delà de ses analyses. Comprendre un auteur, c'est également comprendre ce qu'il rend possible, comprendre les horizons qui s'ouvrent à partir de lui avec la radicale désubjectivisation de Dieu qu'il effectue, même si cela a lieu malgré lui, et même parfois en dépit de ses réserves et de ses interdits. Comme Husserl a pu le mettre en évidence, le présent est le temps premier, origine de 
la temporalité, et les analyses de Husserl sur l'idée de Dieu doivent être interrogées à partir du présent des études phénoménologiques. Cela n'induit pas de relativisme car la question n'est pas intra-historique et tient au fait que le sens lui-même est historique : le passé ne peut avoir un sens que par rapport au présent d'une pensée qui se cherche. Là encore il y a une loi d'essence de l'analyse philosophique: seul celui qui s'interroge aujourd'hui sur Dieu, sur sa phénoménalité, peut dans un acte de reconnaissance philosophique ${ }^{4}$ être à l'écoute de la compréhension historique que Husserl a pu avoir de Dieu. Toute histoire philosophique vit de la question elle-même, de la lumière d'une recherche toujours en cours, ou bien n'est que bavardage.

Cela dit, même dans ce qu'elle ouvre la philosophie de Husserl dessine un style bien précis, et c'est pourquoi il y a également des raisons proprement philosophiques pour étudier la question de Dieu dans la pensée de Husserl. Par exemple on peut noter qu'elle ne fut pas déterminante pour la philosophie existentielle telle que Jean Wahl a pu la développer, et cela parce que la question de Husserl demeure strictement épistémologique, celle de la constitution de l'être de Dieu. Selon l'auteur de La philosophie comme science rigoureuse, les thèses décisives sont d'abord gnoséologiques et ensuite elles peuvent recevoir une signification axiologique et pratique. Ainsi, de façon rigoureusement philosophique, Husserl ne décrit pas la relation de l'homme à Dieu comme une relation d'étant à étant, mais comme la relation de l'être absolu du sujet à son propre idéal et donc comme une transcendance interne à l'immanence de la conscience. Autrement dit, la réduction phénoménologique conduit en deçà de la distinction ontique, comme dirait Heidegger, entre la personne humaine et la personne divine, et elle permet ainsi d'élucider le sens d'être de Dieu pour toute subjectivité comme préalable juridique de toute théologie. La perspective adoptée par Husserl est donc strictement ontologique, et c'est ce qui différencie sa philosophie de toute métaphysique reconstructive dont les représentants actuels ne manquent pas. Tout processus d'abstraction passe nécessairement à côté de la connaissance de Dieu à partir de lui-même, et, ici comme partout en phénoménologie, la rigueur consiste à se laisser guider par l'expérience, sans céder au chant des sirènes de l'abstraction. De même que le temps n'est pas une image produite par l'esprit mais une réalité qui se donne à la conscience dans la rétention, Dieu n'est pas lui-même une simple image et il s'agit de sauver sa «réalité » en montrant qu'il peut faire l'objet d'une intuition. La tâche propre de la philosophie comprise comme phénoménologie est de donner à voir Dieu comme on ne l'a pas encore vu, à savoir comme uns structure de la subjectivité. C'est à une telle condition que l'Idée

\footnotetext{
${ }^{4}$ Au double sens envisagé par Jean-Louis Chrétien dans Reconnaissances philosophiques, Paris, Editions du Cerf, 2010.
} 
de Dieu peut être autre chose qu'un invariant anthropologique qui accompagnerait de fait chaque culture et chaque subjectivité.

Comme Husserl le met en lumière dans les Idées I, la force de la réduction phénoménologique à la pure donnée de Dieu est de libérer de toute expérience mondaine de Dieu. Par la mise entre parenthèses de toute considération de Dieu comme un étant rencontré dans le monde et dans l'histoire, il devient possible de le comprendre comme un sens relatif à la subjectivité transcendantale. Une telle démarche possède aussi une dimension libératrice, puisque l'hypothèse de l'anéantissement du monde envisagée au $\S 49$ des Idées I montre comment tout dieu lié au monde serait nécessairement emporté par le monde. Les images construites de Dieu ne manquent pas, mais elles sont radicalement contingentes et la question de la philosophie est de savoir s'il y a un sens de Dieu au-delà de cette relativité. Ainsi la réduction peut donner à voir l'être absolu et nécessaire de Dieu, mais comme Idée constitutive de toute conscience qui ne peut pas disparaître, même si elle peut être oubliée. La réduction phénoménologique conduit donc dans un tout autre lieu que celui de l'anthropologie religieuse et que celui de la théologie, dans la mesure où il s'agit de comprendre le sens de Dieu en tant que tel pour la conscience. Dans son exigence absolue d'une absence de présupposés, la philosophie ne peut être ni une anthropologie, qui présuppose le sens de l'être-homme, ni une théologie avec ses présupposés dogmatiques. Ainsi la réduction, qui n'est pas une exclusion, permet d'éviter de se laisser enfermer dans toute une série de faux problèmes, dans la mesure où il ne s'agit pas d'accéder à un a priori abstrait et construit à partir de la confrontation des différentes religions, ou à partir d'une réflexion sur la fonction sociale de la religion, ou encore d'une étude du sacré telle que l'anthropologie peut la développer.

\section{Accéder au phénomène de Dieu.}

Même dans l'étude d'un auteur il est impossible de dissocier l'interrogation historique et l'interrogation spéculative, qui vivent l'une par l'autre, et le souci constant de ce livre fut, en établissant le dossier de la question de Dieu chez Husserl, de montrer en quoi il s'agit bien d'une interrogation sur l'intentionnalité de la conscience qui ne cesse d'être une énigme. Si la question du temps demeure le cœur de la phénoménologie de Husserl, parce qu'elle conduit à dévoiler la vie originaire du sujet, la question de Dieu n'est jamais vraiment absente, car elle conduit à mettre en lumière en quoi cette vie est par essence téléologique. A la question de savoir si la réduction phénoménologique, telle qu'elle est mise en œuvre par Husserl, permet d'accéder à la pure phénoménalité de Dieu, à Dieu tel qu'il se donne en lui-même, au-delà de toute condition qui le précède et le prédétermine, la réponse ne peut être que contrastée. D'un 
côté Husserl montre comment le sens de Dieu se constitue pour une conscience, d'un autre côté, la perspective demeure volontairement transcendantale et Dieu demeure un a priori de la subjectivité, qui, même intuitif et idéal, demeure ce qui est obtenu à partir du sujet. On est donc encore très éloigné ici de la phénoménologie de la donation telle que Jean-Luc Marion a pu la développer dans Etant donné et qui envisage Dieu comme celui qui se donne sans condition, c'est-à-dire comme une personne qui, au-delà de l'être, aime. Le Dieu de Husserl demeure un Dieu de philosophe au sens d'un principe épistémologique d'auto-détermination du sujet face à ses possibles et comme a priori transcendantal il n'ouvre pas le sujet à une existence à l'impossible dans un oubli, non pas de soi, mais du soi. Cela ne signifie pas pour autant que la confrontation avec le christianisme soit absente, mais elle est en quelque sorte moins pesante que dans les analyses de Kant et celles de Bergson.

Le premier travail phénoménologique est d'accéder au phénomène pur de Dieu, puisque telle est la condition de toute étude historique du concept de Dieu dans la métaphysique et la religion. La réduction phénoménologique permet en effet d'écarter toute idée d'un dieu mondain qui serait la cause empirique du monde, pour s'en tenir à la donnée de Dieu pour une conscience. Il y a là toute une tâche de déconstruction des représentations de Dieu qui conduisent à manquer la réalité de Dieu, qui font de Dieu une fiction de l'imagination ou une fiction conceptuelle, car elles prédéterminent Dieu en fonction de besoins particuliers sans être à l'écoute de sa phénoménalité. Dans ces représentations l'idée de Dieu masque sa phénoménalité et conduit à être aveugle à la constitution de Dieu dans la conscience. Il s'agit néanmoins là d'une véritable épreuve pour la réduction phénoménologique : peut-elle conduire à la pure phénoménalité de Dieu, est-il possible de dire que rien n'est perdu dans une réflexion purement a priori sur Dieu ? Cela est loin d'aller de soi et il est clair qu'il en va avec la possibilité d'accéder au phénomène pur de Dieu de la possibilité même de la phénoménologie. Si en ce qui concerne l'expérience de Dieu il est impossible de s'élever au-delà des considérations psychologiques ou anthropologiques, rien ne pourra attester de la réalité de Dieu pour une conscience et il ne sera qu'un nom attribué à une grande diversité de représentations historiques dans une simple homonymie.

Husserl tente donc d'accéder au phénomène pur de Dieu en mettant entre parenthèses tous les présupposés dogmatiques et cela le conduit à dévoiler Dieu comme une limite idéale, comme un sens idéal. En libérant de cette construction métaphysique qu'est le Dieu fondement du monde, la phénoménologie transcendantale met en évidence que Dieu est une idée que tout homme porte en lui et dont il peut prendre conscience, sans être pour autant une simple idée «produite » par le sujet indépendamment de toute expérience : si l'idée de Dieu s'impose à 
toute subjectivité, cela ne signifie pas que l'origine de l'idée soit dans le sujet. Husserl écrit dans La Crise de l'humanité européenne et la philosophie, «dans le concept de Dieu, c'est le singulier qui est essentiel. Mais ce concept implique, du côté de l'homme, que sa validation de l'être et de la valeur soit éprouvée comme une obligation intérieure absolue ${ }^{5} \gg$. Dieu ne peut se dire idéalement qu'au singulier : si l'histoire des religions met en lumière de multiples dieux, ils ne sont eux-mêmes pensables que sur l'horizon d'un seul Dieu, tout comme la pluralité des mondes n'est envisageable que dans la conscience qu'il n'y a qu'un seul monde possible. Il faut reconnaître que sur ce point le propos de La Crise des sciences européennes et la phénoménologie transcendantale demeure volontairement très classique, et même bien en retrait par rapport aux analyses de son temps, puisqu'il s'agit de concevoir l'idéalisme transcendantal comme l'achèvement du passage historique du mythe à la philosophie. Il faut souligner, encore une fois, que dans cette confrontation de la philosophie à la théologie, il ne s'agit pas de justifier philosophiquement le christianisme, qui n'en a d'ailleurs pas besoin, mais seulement de tenter une pure élucidation de l'idée de Dieu, autrement dit de revenir à une pure expérience de Dieu propre à toute subjectivité, et qui demeure indépendante de toute condition personnelle ou historique, comme elle demeure extérieure à toute grâce divine. Une telle théologie phénoménologique ne part ni du monde, ni des besoins du sujet, ni de l'enseignement que Dieu donne de lui-même par la grâce, et se trouve ainsi sur une ligne de crête difficile à tenir : ne constituer Dieu qu'à partir du pur pouvoir de synthèse de la conscience. Il ne s'agit donc pas de reconstruire Dieu à partir du monde ou à partir des capacités de la subjectivité, ni d'accéder à Dieu à partir de sa parole transcendante, mais de dévoiler l'origine non subjective de l'idée de Dieu dans toute subjectivité, ce qui signifie qu'ici Dieu est relatif au pouvoir d'unification de la subjectivité, et qu'il est même peut-être le pôle idéal de ce pouvoir, sans être pour autant une production du sujet.

Il y a tout de même là une confrontation difficile car tout en reconnaissant que ce pôle idéal de la connaissance, de la vie éthique et de l'histoire peut bien être appelé « Dieu », il n'en demeure pas moins que cet objet, cette valeur idéale, qui est ainsi nommé Dieu par l'idéalisme transcendantal et phénoménologique ne porte ce nom qu'avec difficulté et par simple vicariance. Le chemin de la philosophie qui part du sujet pour aller à Dieu ne peut être parcouru sans danger que dans la conscience que si cet a priori nommé Dieu permet d'approcher de Dieu, il n'est pas le Dieu qui appelle, mais seulement la condition de possibilité du caractère pensable de Dieu. Sans cette structure de la conscience l'appel de Dieu pourrait-il être reçu ? N'est-elle

\footnotetext{
${ }^{5}$ La Crise des sciences européennes et la phénoménologie transcendantale, annexe III, trad. Gérard Granel, Paris, Gallimard, 1976, p. 369-370.
} 
pas le lieu d'une possible conversion? L'appel de Dieu ne vient-il pas bouleverser une conscience qui s'appelle déjà elle-même? La conscience ne doit-elle pas constituer Dieu pour que l'appel de Dieu puisse la reconduire au-delà du possible ?

Ces questions conduisent au cœur des rapports entre philosophie et théologie, et il est tout à fait remarquable que Husserl y soit conduit non par sa formation ou par ses engagements personnels, mais par la contrainte des phénomènes. Husserl part du fait que toute expérience de Dieu demeure inintelligible tant que l'on n'a pas élucidé la relation intentionnelle de l'ego à Dieu. L'a priori subjectif précède l'être de Dieu, mais il s'agit là d'une antériorité transcendantale et non pas empirique. Il est essentiel de distinguer ces deux formes d'antériorité si l'on ne veut pas passer à côté du sens de l'analyse intentionnelle : dire que Dieu est le résultat de ma propre effectuation de conscience ne contient aucun blasphème, si cela est dit du point de vue transcendantal. Or, dans ce qui constitue déjà l'histoire de l'interprétation de la question de Dieu chez Husserl, bien des confusions viennent de l'incapacité à séparer réflexion naturelle et réflexion transcendantale. Ainsi, dire que le sens «moi » est antérieur au sens « Dieu », ne signifie absolument pas que Dieu n'est qu'un produit de notre imagination, et il s'agit, bien au contraire, d'assurer la donnée intuitive de Dieu.

\section{Dieu comme Idée polaire absolue de toute subjectivité.}

Tous les textes de Husserl sur la question de Dieu attestent de sa très grande prudence et de son exigence de s'en tenir à la seule réflexion transcendantale, dont il donne la formulation canonique au $\S 15$ des Méditations cartésiennes. C'est pour cette raison que Husserl ne fait quasiment pas de références à la Bible et qu'il ne fait pas, ou peu, appel au lexique théologique, et sur ce point la différence radicale avec les textes d'Emmanuel Levinas est frappante. Sans vouloir entrer dans la théologie phénoménologique de Levinas, il est juste possible de souligner que c'est sans doute par une nécessité interne à l'analyses intentionnelle que Husserl se refuse à tout détournement du vocabulaire théologique : il ne veut pas confondre manifestation et révélation, ni faire de la manifestation la norme de la révélation. Si la Bible n'est pas un présupposé pour la philosophie, ce n'est pas non plus à la philosophie de dire quelle est la vérité de la Bible. D’une certaine façon dans le rapport entre théologie philosophique et théologie révélée, la posture de Husserl est d'éviter tout asservissement de l'une à l'autre.

En montrant dans le $\S 51$ des Idées I que Dieu ne peut ni être immanent au sens du vécu, ni transcendant au sens du monde, Husserl met en évidence qu'il possède la transcendance de l'Idée, notamment l'Idée d'une connaissance parfaite du monde. Dieu est l'homme infiniment éloigné, une Idée régulatrice, une limite idéale. Il n'est donc pas du tout un simple invariant 
d'une expérience empirique, mais il est un a priori de toute connaissance. Autrement dit, l'autoélucidation de la subjectivité montre qu'il appartient à l'essence de la connaissance d'être polarisée par l'Idée de Dieu et ainsi la réduction phénoménologique dévoile cette Idée comme un a priori de l'autoconstitution du sujet. Dieu n'est donc pas originairement pour la conscience un objet dans le monde, mais il se donne à elle comme un sens idéal et comme l'horizon de toute volonté humaine. Dans cette perspective, il n'est pas question d'intuitionner l'essence de Dieu, de voir Dieu en son essence, mais d'intuitionner l'Idée de Dieu qui s'annonce à la subjectivité comme horizon des horizons.

Si les considérations épistémologiques sont premières, Husserl ne les sépare pas des questions éthiques et l'Idée de Dieu appartient aussi au projet de la purification éthique de la volonté vers une autonomie idéale. Ici encore les quelques analyses esquissées par Husserl possèdent quelque chose de libérateur par rapport aux images de Dieu. D’une façon assez classique par rapport à l'idéalisme allemand, Dieu serait une valeur absolue pour la volonté et non un être que l'on aime et pour lequel on donne sa vie. Certes, Husserl pense qu'il est possible de donner sa vie pour des valeurs absolues, mais ce « donner sa vie » n'a pas le sens qu'il peut recevoir dans le christianisme, car il demeure le projet du sujet pour lui-même. En outre le christianisme n'a rien de commun avec un idéalisme abstrait, car il ne s'agit pas de donner sa vie pour des valeurs, mais pour son prochain et pour Dieu, c'est-à-dire toujours dans un rapport de personne à personne. Il faut reconnaître cependant que Husserl ne décrit pas Dieu comme la seule idée formelle du choix rationnel et qu'il tente de le dévoiler comme l'idée matérielle du devoir absolu. Bien des textes soutiennent l'idée qu'en purifiant l'exigence religieuse il serait possible d'obtenir l'exigence éthique universelle : tout homme éveillé éthiquement doit vouloir des valeurs universelles et éternelles. Cela dit, dans cette éthique phénoménologique Husserl tente de ne pas s'en tenir à Dieu comme a priori construit et abstrait, pour montrer que ce Dieu éthique n'est pas une simple représentation, mais ce qui se donne à la conscience comme un amour des valeurs éternelles, des Idées régulatrices infinies, amour qui fait vivre dans la foi en la raison. Il y a un réel souci chez Husserl, conformément à sa nouvelle compréhension de l'a priori, de dépasser l'alternative destructrice de l'amour pathologique et de l'amour rationnel. Husserl ne revient pas à la description de l'amour comme pure passivité de ce qui me tombe dessus, mais il ne s'en tient pas non plus à la compréhension de l'amour comme acte pur de la volonté sans donnée intuitive. En échappant à ces deux constructions, il s'agit de ne pas passer à côté de la réalité même de l'amour en remontant à sa véritable origine, c'est-à-dire en montrant qu'il relève de bien autre chose que de la synthèse extérieure de l'imagination reliant la donnée d'autrui et la donnée de moi-même. 
On voit ici que pour Husserl la tâche de la philosophie n'est pas de dire sur la transcendance de Dieu la même chose que le christianisme, mais sans l'aide de la Révélation. L’idée de Dieu demeure un horizon infini et cette phénoménalité ne se confond pas avec celle du Dieu chrétien dans les événements de Noël, de Pâques et de la Pentecôte. Dans l'analyse intentionnelle, Dieu demeure un appel que la conscience se donne à elle-même, et cette transcendance appartient bien encore à l'immanence du sujet. Il reste à savoir, ce qui n'est pas selbstverständlich, si la philosophie peut en demeurant elle-même accéder à une transcendance qui n'est plus relative à l'immanence. La conversion philosophique peut-elle empiéter sur la conversion religieuse?

\section{Dieu s'annonce dans l'histoire.}

Les textes sur la téléologie sont de loin les plus nombreux et on a tenté de montrer que ce n'est pas sans raison, car dans cet athéisme méthodologique la téléologie est ce qui assure la dimension intuitive de l'Idée de Dieu, c'est-à-dire, encore une fois, sa réalité. Il est clair également que sur la question de l'histoire la confrontation de la philosophie et de la théologie est particulièrement sensible. Dans le processus d'idéalisation, l'expérience de Dieu devient l'expérience d'un telos qui s'annonce dans l'histoire : Dieu serait l'aspiration secrète de toute l'humanité. Cependant, ce Dieu n'entre pas dans l'histoire pour sauver le monde et l'Emmanuel n'appartient pas à la philosophie.

Tout le travail de Husserl consiste à montrer que l'analyse intentionnelle ne vise pas à construire l'image d'un monde téléologique ; elle se propose au contraire de mettre en lumière en quoi l'évidence de Dieu est l'évidence d'une possibilité, celle de l'unification rationnelle du monde. Le monde peut tendre à devenir un monde de Dieu : «Le problème de Dieu contient manifestement le problème de la raison absolue comme source téléologique de toute raison dans le monde $»^{6}$. Ce que Husserl nomme parfois «la volonté divine » n'a rien ici d'une transcendance radicale qui viendrait me surprendre, mais est la transcendance immanente d'une volonté qui s'éveille à ce qu'elle peut vouloir infiniment. Dans ces analyses Husserl assume totalement le fait qu'il s'agit d'un concept purement ontologique de Dieu. La réduction phénoménologique met entre parenthèses toutes les déterminations ontiques, mais sans envisager un au-delà de l'ontologie. Cela permet de comprendre Dieu comme la vie intentionnelle du monde téléologique en tant qu'il se porte vers son achèvement. Le sens de Dieu comme sens de la vie téléologique donne à comprendre que l'histoire est le sens de l'être

\footnotetext{
${ }^{6}$ La Crise des sciences européennes et la phénoménologie transcendantale, trad. Gérard Granel, Paris, Gallimard, 1976, p. 14.
} 
et l'être du sens et que l'eidos de la subjectivité transcendantale, qui est au fondement de tout étant, ne peut être vraiment élucidée que par l'historicité.

Il n'en demeure pas moins que les textes de Husserl peuvent, à première vue, sembler très décevants selon leur forme, car on ne trouve pas d'analyse suivie sur la question, mais seulement des surgissements du problème de Dieu à propos d'autres thèmes. Sur le fond également le phénoménologue peut demeurer sur sa faim, car les textes paraissent seulement aménager une thèse idéaliste déjà connue. On pourrait alors penser qu'ils ne susciteraient pas l'intérêt du philosophe, pour lequel il n'y a rien de bien nouveau dans cette constitution de Dieu comme Idée depuis Kant, et du théologien pour lequel il n'y a là aucun accès au donné chrétien, ni même un sol commun sur lequel le logos johannique et la raison philosophique pourraient se rencontrer. Cependant tous ces textes, dans leurs hésitations mêmes, qui réunis font sens, sont passionnants pour le présent même de la phénoménologie et de la théologie, car ils dévoilent un chercheur qui ne veut rien lâcher quand il parle de Dieu. Telle est à la fois sa faiblesse et sa force. Cela fait sa faiblesse par rapport à la phénoménologie contemporaine si légitimement attentive à la phénoménalité de Dieu dans sa transcendance propre au-delà de l'immanence ${ }^{7}$. Cependant cela constitue également sa force, car Husserl peut mettre en évidence que Dieu n'est ni une représentation, ni la dernière ombre de la chose en soi. En reconduisant des images de Dieu à Dieu, à sa donnée intuitive, l'idéalisme transcendantal de Husserl peut apparaître comme un moment important de l'étude de la phénoménalité de Dieu et on ne peut que constater que cette phénoménologie a eu la fécondité d'ouvrir des chemins bien au-delà d'elle-même non pour des raisons contingentes, mais parce qu'elle tentait d'appliquer l'exigence de la donnée à Dieu sans laquelle il n'y a pas d'accès à la réalité. Une véritable théologie phénoménologique n'est possible que par la mise entre parenthèses et de l'idée d'un Dieu mondain première cause du monde et de l'idée d'un Dieu subjectif produit par le sujet en fonction de ses facultés.

\section{Une conversion sans confession.}

Néanmoins une deuxième raison fait également la force des essais de Husserl en théologie phénoménologique pure. Plusieurs fois il définit la philosophie comme un chemin non confessionnel vers Dieu, et cela pose la question de la limite du discours philosophique sur Dieu. En effet, la conversion au je transcendantal ne peut pas conduire à une confession et il y a bien une pudeur du phénoménologue qui ne veut pas parler de Dieu à la place de Dieu, qui ne

\footnotetext{
7 Il suffit de penser ici, sans vouloir décrire toute la complexité de ce mouvement, aux œuvres marquantes de Jean-Luc Marion, de Jean-Louis Chrétien, de Jean-Yves Lacoste, qui héritant de Husserl, Heidegger et Levinas, puisent également dans les sources chrétiennes leur analyse philosophique de la phénoménalité.
} 
veut pas non plus parler comme Dieu se parle à lui-même, et qui ne confond pas le langage transcendantal et le langage des anges. La philosophie ne peut reconduire le sujet réfléchissant qu'au philosophe archonte, à cet homme seul en dépit de l'intersubjectivité, qui n'a confiance qu'en lui et qui n'espère qu'en ses propres possibilités. Or si cela libère des liens du monde pour reconduire à la tâche d'être soi en répondant du monde, ce n'est qu'un chemin vers Dieu qui ne conduit pas à Dieu, qui ne peut que préparer en l'homme la possibilité d'entendre la parole de Dieu. Bien évidemment le philosophe sait que l'Idée polaire absolue constituée par la conscience n'est nommée «Dieu » que par un abus de langage, qu'elle n'est qu'un nom-titre qui permet de saisir une direction de sens et de désigner un rapport responsable au sens. D'une certaine façon cela préserve le saut de la foi, de l'expérience proprement religieuse, et il est possible de se demander en retour si cette différence est toujours préservée dans les phénoménologies de l'invisible. Le philosophe peut-il parler du Dieu qui est au-delà de la lumière de l'être sans présupposer la Révélation ? Sans minimiser l'importance de l'apport de la phénoménologie depuis la Seconde guerre mondiale à l'intelligence du phénomène de Dieu, il semble tout de même qu'aucune réduction, qui ne vient pas de Dieu lui-même, ne peut conduire à dire «Toi notre père » ou «fiat mihi », et Husserl nous place au cœur de cette difficulté de la rencontre de la parole philosophique avec une autre parole. Le philosophe qui prend le risque d'aller vers Dieu sans Dieu sait très bien qu'on ne va à Dieu que par Dieu. D'une certaine façon le seul privilège du philosophe transcendantal est de savoir que ce Dieu constitué comme une Idée n'est qu'une idole, et la conscience du caractère inconstituable de l'être de Dieu est déjà un pas important vers l'inimaginable de la Révélation, même si elle n'en est pas une condition.

\section{Les équivoques de la présence.}

Toute la difficulté des textes de Husserl est de vouloir délibérément en rester à ce qu'on pourrait nommer une «théologie profane » qui doit éclairer les structures de la subjectivité, puisqu'elle résulte d'une idéalisation des formes de la vie personnelle, au même titre que les mathématiques sont une idéalisation de l'espace perceptif. Cependant la question phénoménologique se trouve bien là. En effet, Dieu comme Idée est inséparable du moi comme Idée et du monde comme Idée, et c'est bien la conception husserlienne de la manifestation qui bloque tout accès à une obliquité de la manifestation. Au-delà de la séparation qui peut sembler prudente et sage entre la raison et la foi, il faut bien constater que c'est le mode de présence à soi de la subjectivité polarisée par un idéal de transparence et la façon de comprendre le réel comme lui-même polarisé par une donation idéale qui n'ouvrent pas de chemin d'accès vers la 
donnée chrétienne de Dieu. Comme l'a remarquablement montré Jacques Derrida à la fin de son commentaire ${ }^{8}$ de L'origine de la géométrie, Dieu est à la fois celui qui parle dans l'histoire constituée et le pôle de l'historicité de la subjectivité constituante. Cela dit une métaphysique de la présence ne peut écrire qu'une histoire transcendantale dans laquelle l'aujourd'hui de Dieu du christianisme ne peut être pensé. La «présence » de l'Eternel, telle que saint Augustin a pu la décrire, n'a rien de comparable avec celle d'un telos, car il ne s'agit pas de la concevoir, mais de l'aimer. Il serait possible de dire que Husserl demeure fondamentalement grec en faisant du regard eidétique la seule source du salut. Ainsi il ne peut pas envisager la perspective biblique de la conversion du cœur et de l'attente de l'éternité, et même s'il ne confond pas l'Idée de Dieu et Dieu, rien ne permet vraiment de comprendre comment peut s'annoncer ce Dieu qui trouble la conscience depuis l'au-delà du temps. Le Dieu de Husserl demeure un Dieu sans visage pour une subjectivité trop pure pour être concrète. Notamment, dans la purification éthique de la volonté qu'il envisage, à aucun moment Husserl ne se demande comment les valeurs absolues peuvent être reçues pour pouvoir donner lieu à un devoir. Il passe ainsi totalement à côté de la thèse chrétienne selon laquelle toute valeur absolue se donne dans un visage et est reçue dans la faiblesse et la finitude d'une conscience qui découvre son impuissance à se porter elle-même et à dominer le monde. Si l'explication de Husserl avec le christianisme est douloureuse, c'est d'une certaine façon parce que Husserl sait très bien à propos de Dieu (bien moins sans doute à propos du moi et du monde) que sa conception de l'évidence comme trouvant sa source dans la subjectivité transcendantale ne peut pas permettre d'élucider la manifestation de Dieu. Reste à savoir si ce n'est pas toute la compréhension de la manifestation qui doit alors être remise en cause.

Quoi qu'il en soit, si on peut sans doute douter que Husserl parvienne vraiment à accéder à la subjectivité concrète quand on considère les quelques textes sur la question de Dieu, il n'en demeure pas moins que l'apport de Husserl est ici, comme dans bien d'autres endroits, avant tout programmatique. En effet Husserl ne prétend pas renouveler la compréhension de Dieu par la métaphysique, mais avant tout effectuer un changement de méthode. Avant d'interroger les noms divins, le philosophe doit interroger le sens visé par la subjectivité et élucider les modalités concrètes de validation dans l'évidence, afin d'écarter certaines déterminations métaphysiques de Dieu qui demeurent des constructions spéculatives. Nous avons donc bien avec ces textes sur la question de Dieu des prolégomènes pour toute théologie phénoménologique future.

\footnotetext{
${ }^{8}$ Husserl, L'origine de la géométrie, traduction et introduction par Jacques Derrida, Paris, PUF, 1974, p. 162171.
} 


\section{Réponses à quelques objections 9 .}

\section{Un triple combat.}

Pour compléter cette brève présentation de Husserl et l'idée de Dieu, il m'est possible de répondre à certaines objections dont je remercie les auteurs. Il est en effet tout à fait important de montrer en quoi à travers ces textes sur la question de Dieu Husserl est en dialogue réel ou virtuel, notamment en quoi il définit la phénoménologie contre toutes les formes d'objectivisme, de positivisme et de naturalisme qui sont la mort de la pensée parce que le sens n'est plus reconduit à la source vivante du phénomène. En cela il est clair que pour lui tout se décide dans le champ du théorétique. La philosophie est un combat qui, s'il n'a pas lieu les armes à la main, n'en est pas moins violent et pas moins décisif, et dans ce combat contre le positivisme et le scepticisme, qui lui appartient par essence, il en va du sens de l'humanité. Ce combat contre l'absurdité d'une théorie de la connaissance qui part des sciences de la nature ou de la psychologie pour défendre une «philosophie qui part d'en bas », c'est-à-dire des phénomènes, donne sa tension dramatique à l'analyse intentionnelle du sens de Dieu. Dieu n'est pas une curiosité de la philosophie, un objet particulièrement complexe à élucider, mais une question nécessaire de toute recherche a priori au sens vrai du terme. Il reste cependant à savoir, mais ce livre laisse cette question ouverte, si l'héroïsme de la raison défendu par Husserl et le relativisme sceptique ne partagent pas finalement un sol commun de nihilisme, dans la mesure où le sens de la finitude humaine demeure méconnu dans cette conception d'un sujet autocréateur. L'égologie n'est-elle pas contrairement aux apparences l'ultime figure du nihilisme ?

Husserl répond aussi, en effet, aux objections de Heidegger et de Scheler d'avoir manqué la subjectivité concrète, et il est vrai que les analyses du Dieu éthique et du Dieu pôle de la téléologie historique montrent comment concrètement la subjectivité se constitue ellemême en constituant le monde. Il est donc vrai que, d'une certaine façon, Husserl répond ici à l'objection d'en être resté à un « je » abstrait qui ne se temporaliserait pas et ne se constituerait pas dans une histoire. Là encore Husserl veut défendre la spécificité de son idéalisme transcendantal qui ne se laisse pas enfermer dans la caricature d'une subjectivité vide et sans monde par la mise en évidence de Dieu comme Idée qui appartient à l'essence de toute

\footnotetext{
${ }^{9}$ Certaines de ces objections se trouvent dans la recension de Dominique Pradelle, revue Philosophie, n ${ }^{\circ} 114$, été 2012, p. 92-94.
} 
subjectivité. L'expérience de Dieu est l'expérience de soi dans la possibilité de son advenir à soi, mais il est vrai que dans cette démarche purement réflexive on demeure très éloigné d'une herméneutique de la facticité et de la finitude.

Il y a enfin un troisième combat que mène Husserl, celui qu'il mène contre ses élèves et contre les premiers essais de phénoménologie religieuse et là il peut sembler prendre la posture du commandeur qui défend l'orthodoxie de la phénoménologie transcendantale. Certes, le moins qu'on puisse dire est que Husserl ne fut pas très attentif aux tentatives de phénoménologie qui s'éloignaient de lui, néanmoins son souci de justification de sa méthode propre permet d'éviter la rechute dans une perspective ontique qu'il ne s'agit pas de nier, mais qui ne permet pas de s'interroger sur la question de l'origine du sens. Enfin, il serait utile de mettre en lumière l'insertion des propos de Husserl dans un contexte historique bien particulier, notamment pour expliquer un certain anticatholicisme, mais je dois reconnaître n'avoir pas vraiment traité cette dernière question.

\section{Constituer Dieu, n'est-ce pas le perdre ?}

Par rapport à l'histoire de la métaphysique, qui est loin de réduire uniformément Dieu au statut de causa sui, la question classique de l'existence de Dieu est mise entre parenthèses, parce qu'avant de se prononcer sur l'existence ou la non existence de Dieu il est nécessaire de mettre en évidence le sens visé par le mot Dieu. Cette neutralisation de la question de l'existence qui conduit également à suspendre toute réflexion sur l'impossibilité de toute preuve de l'existence de Dieu est tout de même un tournant considérable dans l'histoire de la métaphysique, qui n'est pas pour autant un coup de force évacuant tout un champ de la réflexion philosophique qui fut pourtant central. Au-delà de l'incompréhension dont Husserl fait preuve à l'égard de Descartes sur ce point, au-delà de sa relative ignorance de l'histoire de cette question dans l'ontologie médiévale et moderne, il n'en demeure pas moins qu'il change la manière de poser la question en montrant que la question première est celle du sens et qu'il s'agit d'accéder à un a priori qui se donne dans une intuition eidétique.

La question en effet est de savoir si cette validation du sens de Dieu à partir des exigences de la conscience qui conduit à montrer que Dieu n'est pas absolu au sens où l'ego est absolu peut véritablement être tenue jusqu'au bout. Il faut bien reconnaître que cette absoluité de Dieu demeure relative à une subjectivité qui se pose elle-même et qui donc fixe tout de même les conditions de la manifestation de Dieu. L'accès à l'ego transcendantal qui ne peut ni naître ni mourir ne permet dès lors pas vraiment d'élucider le sens d'une existence comme « créature » relative à un créateur. On peut ainsi légitimement se demander ce qu'il reste de la 
phénoménalité du Dieu créateur dans cette réduction de Dieu à une Idée téléologique. En effet, la réduction phénoménologique dévoile le « je » comme résidu de l'anéantissement du monde et l'Idée de Dieu comme le corrélat du « je », mais dès lors il n'y a pas que le monde qui soit anéantit, mais toute transcendance radicale. Il y a là un double effet de la réduction : si elle libère de toute idée d'un Dieu lié au monde, fondement du monde, et donc pour Husserl causa sui et créateur, elle perd aussi toute possibilité d'envisager une autre lumière du monde que le sujet. J'ai tenté de montrer en quoi le coût de la réduction est ici exorbitant et que déclarer l'impossibilité phénoménologique de penser Dieu comme créateur, c'est nécessairement perdre Dieu. La question est vraiment très délicate. La victoire contre le positivisme est la condition de possibilité d'une pensée de Dieu, mais elle prend ici la forme d'une victoire à la Pyrrhus, car l'idéalisme transcendantal en faisant de Dieu un objet de la conscience perd Dieu comme absolu. J'ai voulu montrer que Husserl n'était pas aveugle à cette difficulté de la phénoménologie transcendantale et y répondait par une séparation de la philosophie et de la religion. La philosophie ne peut qu'élucider Dieu comme sens pensable pour toute subjectivité, sans présupposer que ce sens possède une dénotation, et le propre de l'expérience religieuse serait que Dieu s'y atteste de lui-même et ménage par le don de la foi la possibilité de sa propre manifestation. Elucider l'Idée de Dieu, ce n'est pas donner à voir Dieu, mais c'est tout de même pour Husserl sauver l'humanité de sa misère philosophique en donnant accès au sens de Dieu pour une conscience. La philosophie rend Dieu pensable au-delà de toute facticité, sans vouloir être le dernier mot sur Dieu. Il y a dans cette normativité une réelle difficulté philosophique : en étant libératrice de la naïveté et en disant ce que ne peut pas être Dieu, la philosophie fixe tout de même à la phénoménalité de Dieu des conditions trop contraignantes pour convenir à Dieu. J'ai tenté d'esquisser une solution qui préserve l'apport de cette démarche sans prétendre qu'elle appartient à Husserl : l'homme doit se sauver lui-même en constituant Dieu comme Idée pour que Dieu puisse le sauver par son appel qui transgresse toute condition a priori.

\section{L’athéisme est un intuitionnisme.}

En outre il est manifeste en effet que l'athéisme méthodologique est une conséquence directe de l'exigence d'intuitionnisme selon laquelle c'est l'essence de l'objet qui impose le mode de donation. Or ce mode de donation, par exemple celui de la chose sensible par esquisses, s'impose même à Dieu comme représentant idéal de la connaissance absolue comme Husserl le dit au $§ 150$ des Idées I. Il est certain qu'il s'agit là d'une fiction méthodologique qui consiste à se placer du point de vue de Dieu, pour montrer que chaque type d'objet est le chemin de sa propre connaissance, même pour un sujet idéal. Si Dieu perçoit, il perçoit nécessairement par 
esquisses, néanmoins cela laisse libre de penser que Dieu puisse posséder un autre mode de présence au monde que la perception et qu'il soit en cela bien autre chose que cette idole qu'est le représentant idéal de la connaissance absolue. Il reste cependant à savoir si avec cette nouvelle perspective on ne sort pas de la réflexion transcendantale. Cela conduit alors à nouveau au difficile rapport entre raison et Révélation en ce qui concerne la phénoménologie de l'expérience religieuse. Il y a sur ce point une ambiguïté des textes de Husserl, car pour la plupart ils montrent comment c'est la raison qui élucide les conditions de possibilité de l'expérience religieuse et en quoi la foi en l'Idée est la mesure idéale de la foi religieuse. Cependant, certains textes, à caractère plus autobiographique, soulignent le danger d'une telle réduction de la foi religieuse sans la foi religieuse. Tout son rationalisme montre que la raison éclaire la foi, sans nier pour autant que dans ces analyses la foi doit guider la raison. Husserl ne s'explique pas vraiment sur ce point, et s'il soutient qu'il faut voir pour croire, rien dans sa philosophie ne lui permet de dire qu'il faut croire pour voir. La question demeure donc : une phénoménologie de l'expérience religieuse qui n'a pas lieu sur le fond de la foi ne fait-elle pas de la réduction une suppression?

Il est vrai que contre le scepticisme Husserl distingue la religion comme fait culturel et la religion comme Idée qui dès lors est une religion sans Eglise. Dieu n'est pas une essence qui serait à dégager par variation imaginative, mais une Idée normative limite. Dans ce cadre les critiques d'ethnocentrisme ne portent pas, car il ne s'agit pas de faire d'une religion historique particulière la norme des autres, mais d'élucider a priori le sens de toute religion. Quand Husserl dit que le monothéisme est le telos de toute religion il s'agit seulement de signifier que le mot Dieu ne peut véritablement se dire qu'au singulier, comme Idée polaire absolue, et en un sens le polythéisme lui-même se comprend sur cet horizon idéal. C'est l'idéalisation qui impose l'unicité et non pas une religion historique particulière. Bien sûr la question est complexe dans la mesure où dans bien des textes le christianisme semble être la religion qui accomplit le telos. Husserl cependant tente de montrer que le monothéisme est une Idée que chaque religion porte en elle comme ce qui rend la religion elle-même pensable. Le reproche d'ethnocentrisme tend à ne voir dans le monothéisme qu'une antériorité empirique abusive alors qu'il s'agit d'une antériorité transcendantale qui fixe des conditions de possibilité à la religion. En outre, le refus de toute Idée normative pour la religion doit conclure à l'absence d'une essence de la religion et les religions ne sont plus que des faits historiques irrationnels, ce qui est revenir à une sorte d'ethnocentrisme sceptique. Je ne pense pas que Husserl veuille assurer une position ethnocentrique par la téléologie, même si la question demeure à cause de cette méthode en 
zigzag entre la religion historique et la religion comme Idée qui donne à voir une histoire transcendantale de la religion.

\section{Ethique et religion.}

Enfin, il est vrai que Husserl veut imposer une structure d'horizon éthique à la religion et ainsi faire de l'éthique la vérité de la religion, ce qui conduit à rabattre le sens de l'universel dans la religion au sens de l'universel en philosophie. De ce point de vue, il y a une forme d'anticatholicisme qui conduit Husserl à voir dans la Réforme le telos de l'histoire des religions parce qu'elle serait la religion guidée par l'idée de la philosophie. Cela dit, là encore, Husserl ne dit rien de précis sur la Réforme, il ne cite pas les textes et les thèses principales, mais s'en tient à l'idée qu'elle serait une percée de la liberté et qu'elle serait ainsi un moment important dans l'accomplissement du telos de l'humanité qui demeure celui de la philosophie. L'unique Idée normative, y compris pour la religion, est l'Idée de philosophie. En réalité le projet de Husserl est de montrer qu'avec la Réforme la religion commence véritablement à s'arracher à la particularité historique pour ne dépendre que de l'esprit seul, mouvement qui conduit la religion à s'achever en éthique. Le monothéisme n'est pas une religion particulière cherchant à dominer les autres quand, idéalisé, il devient une instance critique qui délivre du dogmatisme. Ainsi le monothéisme se trouve lui-même idéalisé par Husserl et prend la signification d'un audelà des religions historiques, d'un au-delà de l'idolâtrie. En outre, puisque cette téléologie à un horizon éthique, c'est finalement l'éthique elle-même qui est le monothéisme et cette idée va se retrouver, certes sous une forme sensiblement différente, dans la pensée de Levinas. Là encore, la perspective transcendantale sur la religion se paye cher car jamais Husserl ne semble douter que l'esprit de la raison libre, qui devient avec la Réforme l'esprit de la modernité, ne soit la propriété de la philosophie, et ainsi jamais il n'envisage la signification proprement théologique de l'esprit comme don. Cette religion idéalisée qui doit certes n'être qu'une instance critique n'est plus une religion.

Entre la simple anthropologie religieuse qui demeure prise dans le relativisme et la critique philosophique de la religion qui ne peut fixer que des conditions négatives a priori, la phénoménologie de l'expérience religieuse doit encore trouver son chemin, notamment par une herméneutique de la facticité et de la finitude. J'ai seulement défendu l'idée qu'en ne déterminant plus Dieu à partir du « représenter» comme activité originaire de l'homme et en revenant à la donnée première de l'homme par rapport à laquelle toute représentation est dérivée, Husserl à la fois ouvre ce chemin d'une véritable phénoménologie religieuse et ne s'y engage pas, car Dieu est encore éclairé à partir des exigences du sujet et non à partir de sa seule 
donnée, même s'il n'est pas une production du sujet. Il reste bien sûr à savoir si cette pure donnée de Dieu par lui-même ne relève pas seulement de la grâce et si elle peut être reçue sans défaire les attentes du sujet à l'égard de Dieu. Ne faut-il pas viser Dieu comme Idée pour que la conversion à partir de la manifestation que Dieu lui-même ménage en nous soit possible ? Ne faut-il pas chercher l'évidence des valeurs absolues pour que l'évidence du Dieu qui aime et qui fonde toutes les évidences puisse être reçue en transfigurant ces valeurs ?

\section{Réponses à Jérôme de Gramont}

\section{Husserl et la Bible.}

Il est indéniable qu'il y a une tension interne à la phénoménologie de Husserl, soulignée par de nombreux commentateurs, qui tient il est vrai à des raisons différentes, mais finalement convergentes. La téléologie historique envisagée par Husserl dans La Crise des sciences européennes et la phénoménologie transcendantale n'envisage que la foi en la science, la foi en la raison, et dans cette histoire de l'Europe la Bible n'a pas de place : c'est Thales et non Moïse qui est le père originaire de l'humanité. Cette absence totale de la Bible et de la philosophie médiévale dans cette œuvre qui n'évoque la religion que sur la forme du mythe, qui ne relève pas encore de l'histoire de la raison, ne correspond pas à une négligence, mais tient au fait que pour Husserl la Bible n'appartient pas vraiment à l'histoire de l'intentionnalité. Bien évidemment, et d'autres textes le confirment, il y a du sens dans la Bible, mais toute cette histoire des religions ne peut devenir intelligible qu'à partir de la pure histoire du sens dont la phénoménologie transcendantale est le projet secret. Pour le dire un peu brutalement, la Bible n'est pas un moment incontournable de l'étude de l'énigme du sens et il faut d'abord dissiper cette énigme pour pouvoir rendre raison de la Bible. A aucun moment Dieu n'est considéré comme une évidence d'un autre type qui serait la condition de toutes les évidences, et c'est toujours l'évidence du « je » qui est la source de toutes les évidences. Dans ces conditions, il est impossible de passer de la foi en la raison à la foi en Dieu sans rabattre la seconde sur la première.

Il n'en demeure pas moins que Husserl est un vrai chercheur qui ne triche jamais avec les phénomènes et qui inlassablement se confronte avec ses propres apories, selon cette thèse méthodologique implicite qui sert de guide dans la recherche, à savoir que toute aporie est 
l'indice d'un reste de construction et donc du non accès à la pure phénoménalité. Cette thèse de Jean-Yves Lacoste que vous avez citée, à savoir que l'enseignement de la réduction est qu'il y a de l'irréductible ${ }^{10}$, a effectivement guidé ma lecture de Husserl. Plusieurs fois dans son œuvre Husserl se heurte à de l'irréductible ; on peut penser à l'irréductibilité de l'impression originaire et le fait que pour dire la pure mouvance du temps, même le terme de flux est inadéquat et que les noms adaptés au temps constitué ne permettent pas de dire le temps constituant. On doit penser également à l'irréductibilité du je transcendantal, cette étrange structure de la transcendance dans l'immanence, qui a tant fasciné Levinas et qui porte toute l'énigme d'une subjectivité à la fois constituante et constituée par ce qu'elle fonde. Il ne faut pas oublier les textes sur le monde comme horizon de tous les horizons. Certes à aucun moment Husserl ne veut conclure que ce sont des apories de la chose même, car ce serait pour lui un argument paresseux qui met fin à l'analyse intentionnelle. Dieu, comme tout ce qui relève d'une singularité absolue, peut lui aussi apparaître comme un irréductible et Husserl sait bien que Dieu «dans les limites de la simple égologie », pour reprendre votre expression, n'est pas vraiment Dieu et que les mots ne la conscience constituante réduisent Dieu à un objet immanent. Il est en effet impossible de laisser le dernier mot à Dieu dans une telle perspective. Néanmoins, j'ai effectivement voulu montrer que les différents textes sur la question de Dieu laissent apparaître une tension interne à la phénoménologie de Husserl, puisque tout en montrant les différentes voies de la constitution de Dieu, Husserl ne ferme pas la porte à l'irréductibilité de Dieu aux actes de la conscience constituante. Il y a ici une analogie possible avec les apories de la conscience du temps : l'ego est antérieur à Dieu parce qu'il est la source ultime du sens, mais l'ego lui-même ne peut constituer Dieu qu'en tant qu'il nous touche déjà d'une façon non conscientielle. Cette tension interne est bien le lieu d'une explication difficile avec la Bible, au moins pour une théologie phénoménologique qui ne se veut pas purement formelle: la philosophie part de la donnée du sujet à lui-même pour accéder à la donnée de Dieu comme Idée, la théologie, elle, part de la donnée de Dieu comme appel pour accéder à la donnée du sujet comme répondant. En philosophie on répond de Dieu en répondant à soi, en théologie on répond de soi en répondant à Dieu.

\section{Husserl et Kant.}

Il y a effectivement comme vous le dites un modèle kantien et fichtéen dans toute cette interrogation sur la question de Dieu et j'ai tenté une vraie confrontation, notamment avec Kant.

\footnotetext{
${ }^{10}$ Jean-Yves Lacoste, La phénoménalité de Dieu, Paris, Editions du Cerf, 2008.
} 
Il resterait à affiner encore cette perspective génétique en montrant comment Husserl a reçu Kant par le néokantisme, mais mon souci était surtout de montrer en quoi une compréhension très différente de l'a priori conduit à des analyses très différentes de l'Idée de Dieu. Bien évidemment le piège de toute confrontation tient à l'écart inévitable, et parfois considérable, entre ce qu'à dit effectivement un auteur et l'image qu'en possède celui qui veut le dépasser. Même si Husserl ne cesse de se confronter à Kant qu'il a vraiment lu, même si toute son œuvre est une réinterprétation de La Critique de la raison pure, il est manifeste qu'il laisse de côté bien des aspects de la philosophie de Kant. Il resterait bien sûr à faire le délicat partage entre ce qui relève de l'historicité de toute interprétation et ce qui dépend de la finitude d'un auteur. Quoi qu'il en soit, Husserl nous intéresse même quand ce qu'il dit de Kant ne correspond pas à notre compréhension actuelle de Kant. Mon expression à propos du Dieu éthique d'un « reste de kantisme en philosophie » est une reprise d'une proposition de Heidegger dans son autointerprétation et réduit effectivement Kant à un nom-titre d'un idéalisme abstrait, ce qui ne lui rend pas justice.

Comme l'a indiqué très justement le père Philippe Capelle lors de son introduction à cette séance, Heidegger est le grand absent de ce livre, et cela d'une façon tout à fait volontaire. Il s'agissait d'une part de ne pas écraser les quelques analyses de Husserl sur la question de Dieu sous le poids des textes beaucoup plus conséquents de Heidegger et, d'autre part, d'interroger Husserl à partir de lui-même et non pas à partir d'une tout autre position de la question. Cela dit ce grand absent est également nécessairement très présent, notamment quand il s'agit de comprendre comment le «kantisme » est une construction intellectuelle qui bloque l'accès à la phénoménalité. Kant n'est pas nécessairement ici dans Kant, et il n'est que le nomtitre d'une attitude théorique selon laquelle la distinction de l'attitude naturelle entre le monde pour moi et le monde en soi n'est pas véritablement et pleinement abolie. Pour forcer le trait, il serait possible de dire qu'un phénoménologue n'est jamais assez anti-kantien, puisqu'il doit retourner aux choses mêmes, ce qui n'est pas, bien évidemment, rendre justice à la richesse et à la complexité des analyses kantiennes, dont on est loin d'avoir épuisé la nouveauté. Il serait en outre tout à fait possible de montrer que Kant ne serait pas sans réponse aux critiques de Husserl et que sur la question de Dieu il ne tombe pas dans les difficultés auxquelles s'exposent Husserl dans la transformation éthique de Dieu.

\section{La phénoménologie à l'épreuve de ses limites.}

Vous citez enfin l'idée de Ricœur selon laquelle il y aurait des voies frayées, puis abandonnées dans la phénoménologie de Husserl. Je préfère votre expression de « modèle » et 
l'idée qu'il y aurait un modèle kantien, un modèle aristotélicien et, de façon intempestive, un modèle lévinassien dans l'interrogation sur Dieu. En effet, cette distinction trop scolaire des voies, que Husserl utilise certes dans le cours de Philosophie première, ne rend pas compte de l'unité et de la persévérance de l'interrogation eidétique husserlienne. En outre ce qui fait la tension interne de la pensée de Husserl sur Dieu est qu'il n'y a pas de voies abandonnées : les perspectives gnoséologique, éthique et téléologique se complètent l'une l'autre dans l'étude de l'Idée de Dieu en tant qu'elle anime la vie de la conscience, et y voir un dilemme ce serait, comme le souligne Derrida, retomber dans une attitude spéculative. Le style propre de Husserl est de ne rien lâcher, même dans ses rétractations, et de compléter sans cesse, d'affiner sans cesse, ses analyses. Si on peut voir apparaître dans certains textes un Husserl quasi mystique, il ne s'agit en rien de l'abandon par un philosophe âgé de la voie gnoséologique.

Effectivement, avec la question de Dieu la phénoménologie est à l'épreuve de ses limites, même si ce n'est pas le seul lieu où elle le soit. J'ai voulu montrer qu'au-delà de l'accusation d'idolâtrie Husserl ne fait pas que transformer Dieu en objet et qu'il y a dans la façon dont il envisage la transcendance de Dieu une question nécessaire. Il y a ici deux choses à distinguer. D'une part il ne faut pas rigidifier la pensée de Husserl en une doctrine clairement identifiable dans un chapitre d'un livre scolaire, et il convient de la rendre à sa source vivante qui est la contrainte des phénomènes, puisque c'est bien elle, comme vous le dites, qui permet d'envisager toutes les possibilités de la donation. D'autre part, Husserl, dans la tension de sa confrontation avec le christianisme, enseigne le saut inévitable entre la donnée de Dieu à toute subjectivité et la donnée du Dieu chrétien. Si bien évidemment la phénoménologie peut envisager un Dieu qui se donne en excès, elle ne peut l'envisager que comme une possibilité et ne peut pas aller plus loin. C'est aussi en cela que les textes de Husserl peuvent être une source de réflexion pour la phénoménologie contemporaine de la donation de Dieu et n'est pas qu'un exemple de plus dans la longue liste des manquements de la phénoménalité de Dieu.

Il est tout à fait vrai qu'il y a deux manières de lire ces textes de Husserl sur la question de Dieu. Soit on les lit de façon négative, restrictive, en n'y voyant qu'une nouvelle expression d'une philosophie de la volonté pure, d'une ontothéologie, d'une métaphysique de la subjectivité, d'une idolâtrie du moi, et donc comme appartenant à un passé révolu de la pensée, soit on comprend ce passé de façon vivante à partir de l'avenir de la phénoménologie et on peut effectivement voir aussi dans ces textes comment la phénoménologie est à l'épreuve de ses propres possibilités et comment le principe des principes selon lequel l'intuition est la seule source de droit permet de s'interroger sur l'être de Dieu à partir de sa seule phénoménalité. 
